\title{
Research on Efficient Toll Plaza Model
}

\author{
Xuan Wang \\ School of Department of Mechanical Engineering, North China Electronic Power University, Baoding \\ 071000, China
}

\begin{abstract}
Toll plaza is not only an important part of the toll station, it's also vital for vehicles in and out of the highway. According to international experience, in the case of effective management (the number of equipment is reasonable, and equipment is relatively simple), the cost and income ratio as follows: $5 \% \sim 10 \%$. If the number of toll facilities is greater than the required number of operating, the cost and income ratio increased to $10 \% \sim 15 \%$. If the management is poor, the cost and income ratio may exceed $30 \%$. So setting up reasonable toll plaza size and the number of lanes should be the focus of the toll booth design. The ultimate aim of toll plaza design is to maximum to avoid the occurrence of traffic accidents and traffic congestion situation, and make the vehicle through a toll plaza safely after entering the lane to accept service. Of course, vehicles also require enough space for payment and going back to the normal track. In order to providing a safe, comfortable, and efficient work environment for management.
\end{abstract}

Keywords: Model, Toll Plaza, M/G/K Algorithm.

\section{Introduction}

For generality, we set the volume of traffic, service time and level of service as variables. Then, we try to model the traffic capacity. On the one hand, the model needs to show the basic traffic capacity. On the other hand, it is required to depict the distribution under the influence of traffic flow increase and decline. Then we can change the inputs to do some simulations. Only using many simulation results can we build a relationship between input and output of the complicated transportation system.

In this section, firstly, we state the principles of the designing of Toll Plaza. Then, we calculate the specific input and output of the complicated transportation system by $\mathrm{M} / \mathrm{G} / \mathrm{K}$ algorithm. In this part, we anglicized the traffic capacity of MTC and ETC. Then, we come to the traffic capacity of the Toll Station. Finally, we get the number of different lanes and the linear of Toll Plaza.

\section{Model for Toll Plaza}

\subsection{The Principle of Designing Toll Plaza.}

The design of the toll plaza should follow the following principles:

Toll plaza should have enough charge lanes and parking space for the use of rush hour traffic.

Toll plaza shall not become an obstacle to the security, and avoiding the toll plaza on the interchange ramp affects the mainline traffic.

Toll plaza should be set as far as possible on the flat and straight line sections, for convenient parking and start of the vehicle.

Toll plaza construction should provide convenient conditions for billed business and management business.

\subsection{M/G/K Algorithm.}

\subsubsection{Traffic Capacity Analyses (Table 1)}

Table. 1 reduction factor of the vehicle

\begin{tabular}{|llll|}
\hline Vehicle type & Small car & Middle car & Large car \\
\hline reduction factor of MTC & 1.0 & 1.7 & 2.0 \\
reduction factor of ETC & 1.0 & 1.3 & 1.7 \\
\hline
\end{tabular}




\subsubsection{The Traffic Capacity Of MTC}

$$
C_{\mathrm{b}}=\frac{3600}{T_{S}+T_{G}}
$$

$C_{b}$ : The traffic capacity of MTC

$T_{s}$ : The standard car service time.

$T_{G}$ : The standard cars leave time

It is very simple for vehicles to enter the toll station through the entrance. The service time obey normal distribution basically; however, as it is need to pay cost at the export and service time under the influence of many factors that it obey random distribution generally; and the leave time obey normal distribution in general.

\subsubsection{The Traffic Capacity of ETC (Table 2)}

Design aligned with the main line

$$
\begin{aligned}
& C_{S}=\frac{1000 v_{0}}{l_{0}} \bullet f_{1} \bullet f_{2} \\
& l_{0}=\max \left\{l_{r}, \frac{v_{0} t_{0}}{3.6}+\frac{v_{0}{ }^{2}}{254 \phi}+l_{\mathrm{c}}+l_{\mathrm{a}}\right\}
\end{aligned}
$$

$v_{0}$ : driving speed

$l_{c}$ : The average length of car (A car usually take $6 \mathrm{~m}$.)

$l_{a}$ : The safe stopping distance between vehicles (Usually take $2 \mathrm{~m}$ ).

$\phi$ : The longitudinal adhesion coefficient.

$f_{1}$ : Transverse interference coefficient $\left(0<f_{1}<1\right)$. Caused by the charge lane width, island, charging device and prompt device toll station fixed structure.

$f_{2}$ : Correction factor $\left(0<f_{2}<1\right)$.

Table 2 The relationship between the longitudinal adhesion coefficient and vehicle speed

\begin{tabular}{l|llllllll}
\hline$v(\mathrm{~km} / \mathrm{h})$ & 120 & 100 & 80 & 60 & 50 & 40 & 30 & 20 \\
\hline$\phi$ & 0.29 & 0.30 & 0.31 & 0.33 & 0.35 & 0.38 & 0.44 & 0.44 \\
\hline
\end{tabular}

\subsubsection{The Traffic Capacity of the Toll Station}

$$
C=C_{m}^{M T C}+C_{n}^{E T C}
$$

M: The number of MTC lanes.

$\mathrm{N}$ : The number of ETC lanes.

\subsubsection{The Calculation of the Number of MTC Lanes}

$D H V_{M T C}=A A D T_{M T C} \times K_{M T C} \times D_{M T C}$

$D H V_{M T C}:$ Standard design hourly volume

$A A D T_{M T C}$ : Average daily traffic volume.

$K_{M T C}$ : Design hour traffic flow coefficient, standard value is 0.12 , value range: suburbs are Average of $0.085 \sim 0.085$, near highway is $0.12 \sim 0.15$

$D_{M T C}$ : Uneven distribution coefficient in the traffic flow direction, standard value is 0.6 , the range is 0.5 0.6;

The average of vehicle arriving at: 
$\lambda=D H V_{\text {MTC }} / 3600$

Toll station traffic intensity:

$\rho=\lambda / \mu$

Each lane's traffic intensity:

$\eta=\frac{\lambda}{K \times \mu}$

$K:$ The number of lanes

$\mu$ : Average service intensity of each lane (The reciprocal of the average service time).

Apparently, lane service rate is less than the average arrival rate of each lane when $\eta \geq 1$, such vehicles would not be handled in time that driveway lined up to loop indefinitely. Consequently, under the condition of $\lambda, \mu$ are certain, we should determine $\mathrm{K}$ according to $\eta \leq 1$. In this case, can the vehicles that arrived at the toll plaza pass the toll lane after average waiting time. Take boundary condition as $\eta 1$, and then we can calculate the number of lanes $m$.

\subsubsection{The Calculation of the Number of ETC Lanes}

Design the limit velocity V of the ETC lanes based on road level design, regional transportation development, the service condition of ETC in the region and ETC drivers' overall quality etc.

Solve the model on the basis of the design of ETC and traffic capacity. Calculate the basic capacity $C_{s 0}$ of an ETC lane.

Based on the specific volume of predicted ETC designed hourly traffic volume $D H V_{E T C}$ and $C_{S O}$ to estimate the least number of lanes needed, $K^{\prime}, K=D H V_{E T C} / C_{s 0}$.If $K^{\prime}$ is an integer, $n=K$. Otherwise, take $K^{\prime}=[K]+1$ generally.

Double calculation capacity. Based on the ETC lane the location of the simulation, prediction of traffic volume, traffic composition, Lane width design and other parameters to simulate the actual traffic capacity $\dot{C}_{S}^{\dot{E T C}}$, if $\dot{C}_{S}^{\dot{E T C}} \geqslant D H V_{E T C}$, that meet the requirements, designing the number of lanes $n=K^{\prime}$; if not, increase the number of lanes $K_{l}$, take the value of $K_{l}$ as:

$$
K_{1}=\frac{D H V_{E T C}-C_{S}^{E T C}}{C_{S 0}}
$$

To double simulate the actual traffic capacity $\ddot{C}_{S}^{\ddot{E T C}}$ of composite toll again, if $\boldsymbol{C}_{S}^{\ddot{E T C}} \geqslant D H V_{E T C}$, that meet the requirements. Then to design the number of lanes $n=K_{l}+K^{\prime}$. If not, increase $K_{l}$ continuously, repeating the process above until meet requirements. Get the final result:

$K=K^{\prime}+\sum_{i=1}^{n} K_{n}$

\section{Summary}

The more creative work is that we determine the specific use countermeasures. We find that the traffic flow is inconstant. You may decrease the number of opening the driveway when the traffic flow is small. The number of ETC Lane is 4; the entrance of the MTC Lane is 6; the exit of the MTC Lane is 10 . On the contrary, we design the portable charging machine when the vehicle flow is heavy. The number of ETC Lane is 6; the entrance of the MTC Lane is 9; the exit of the MTC Lane is 15. The increasing number of the entrance of the MTC Lane is 2; the exit of the MTC Lane is 3 In addition, the portable charging machine can be used when happen unexpected situation. 


\section{References}

[1] Muamer Abuzwidah, Mohamed Abdel-Aty, "Safety assessment of the conversion of toll plazas to all-electronic toll collection system, Accident Analysis \& Prevention, "Volume 80, July 2015, Pages 153-161, ISSN 0001-4575.

[2] M. Papageorgiou, I. Papamichail, A.D. Spiliopoulou, A.F. Lentzakis, "Real-time merging traffic control with applications to toll plaza and work zone management, Transportation Research Part C: Emerging Technologies, "Volume 16, Issue 5, October 2008, Pages 535-553, ISSN 0968-090X.

[3] Seongmoon Kim, "The toll plaza optimization problem: Design, operations, and strategies, Transportation Research Part E: Logistics and Transportation Review, "Volume 45, Issue 1, January 2009, Pages 125-137, ISSN 1366-5545.

[4] L. He, Y. Wu, X. Zeng and A. Yang, "The design of toll plaza using the combination of modeling and simulation," Proceedings of 2011 IEEE International Conference on Grey Systems and Intelligent Services, Nanjing, 2011, pp. 744-747.

[5] J. g. Qiao, C. y. Wang and A. r. Li, "Research on number of lane at toll stations of freeway mainline," 2011 International Conference on Electric Technology and Civil Engineering (ICETCE), Lushan, 2011, pp. 5342-5345.

[6] J. Xing, H. Takahashi and H. Kameoka, "Mitigation of expressway traffic congestion through transportation demand management with toll discount," in IET Intelligent Transport Systems, vol. 4, no. 1, pp. 50-60, March 2010. 